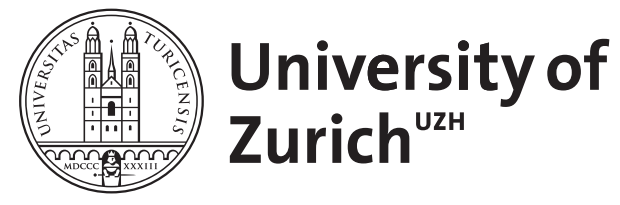

Zurich Open Repository and Archive

University of Zurich

University Library

Strickhofstrasse 39

CH-8057 Zurich

www.zora.uzh.ch

Year: 2013

\title{
Patienten verstehen Medikationsveränderungen während der Hospitalisation
} nicht immer

Markun, Stefan

DOI: https://doi.org/10.1024/1661-8157/a001247

Posted at the Zurich Open Repository and Archive, University of Zurich ZORA URL: https://doi.org/10.5167/uzh-78332

Journal Article

Accepted Version

Originally published at:

Markun, Stefan (2013). Patienten verstehen Medikationsveränderungen während der Hospitalisation nicht immer. Praxis, 102(8):487-488.

DOI: https://doi.org/10.1024/1661-8157/a001247 


\title{
Patienten verstehen Veränderung von Medikamenten während der Hospitalisation nicht immer
}

\author{
Frage: \\ Welches ist die Häufigkeit von vermutlichen Ärztefehlern bei Medikamentenverordnungen und \\ wie hoch ist die Prävalenz von Missverständnissen bezüglich Änderungen der Medikation bei \\ Patienten nach Spitalaustritt.

\section{Hintergrund:} \\ Häufig werden nach Spitalentlassung neu etablierte medikamentöse Therapien weitergeführt \\ und häufig werden bestehende Therapien während der Hospitalisation geändert oder gestoppt. \\ Eine korrekte Applikation dieser medikamentösen Behandlung ist insbesondere nach \\ Spitalaufenthalt entscheidend. Mögliche Fehlerquellen in der Anwendung der Medikamente \\ bestehen in Verschreibungsfehlern auf Ärzteseite und in Missverständnissen auf Patientenseite. \\ Vermeidbar wären auf Ärzteseite viele Verschreibungsfehler durch korrekten Abgleich der \\ Medikationslisten. Die kumulierte Häufigkeit von Verschreibungsfehlern und \\ Missverständnissen wurde bisher nach Spitalaustritt noch nie evaluiert.
}

\section{Einschlusskriterien:}

- Männer und Frauen über 65 Jahre alt

- Spitaleintritt mit Symptomen eines akuten Koronarsyndroms, Herzinsuffizienz oder Pneumonie

- Englisch oder Spanisch sprechend

\section{Ausschlusskriterien:}

- Patienten in palliativer Pflegesituation

- Unfähigkeit des Patienten oder einer Betreuungsperson für ein Telefoninterview oder verwirrter, deliranter Eindruck während des Telefoninterviews

- Kognitive Einschränkungen mit Versagen im Uhrentest

\section{Studiendesign und Methode:}

Prospektive Kohortenstudie

\section{Studienort:}

Yale-New Haven Hospital, New Haven (Connecticut), USA

\section{Outcome:}

- Exaktheit des Medikamentenabgleichs bei Spitalaustritt verglichen mit Spitaleintritt

- Verständnis der Patienten bei durchgeführten Medikamentenänderungen

\section{Resultat:}

- Daten von 377 Patienten konnten in der Studie ausgewertet werden. Das mittlere Alter betrug 77 Jahre, 54\% der Patienten waren männlich, 82\% der Patienten hatten weisse Hautfarbe.

- Bei 52\% der Patienten bestand bei Eintritt ein akutes Koronarsyndrom, bei 39\% eine Herzinsuffizienz, bei 24\% eine Pneumonie. 14\% der Patienten hatte mehr als eine dieser Diagnosen. 
- Bei Eintritt wurden 2534 Medikamentenverschreibungen gezählt, bei 22\% dieser Verschreibungen wurde bei der Entlassung eine Dosisanpassung festgestellt, oder das gänzliche Absetzen des Medikamentes. Im Rahmen dieser Änderungen wurde in 24\% der Fälle ein Arztfehler vermutet. Signifikant gehäuft im Vergleich traten diese vermutlichen Fehler bei Therapien auf, welche unabhängig von der Eintrittsdiagnose waren.

- Bei den Befragungen über die Medikamente nach der Entlassung (nur als korrekt angenommene Verordnungen eingeschlossen) zeigte sich, dass die Patienten $69 \%$ der Dosisänderungen nicht korrekt verstanden hatten, dies galt ebenso für $82 \%$ der gestoppten Medikamente, sowie für $62 \%$ der neu verordneten Medikamente.

- Insgesamt zeigte sich, dass in 81\% der Fälle die medikamentöse Therapie entweder durch vermutete Verschreibungsfehler, oder durch Missverständnisse kompromittiert war.

\section{Kommentar:}

- Bezüglich der Verschreibungsfehler liefert die Studie nur eine mutmassliche und auf Änderungen selektierte Auskunft. Die wirkliche Adäquatheit und auch Vollständigkeit der indizierten Therapien wurde zu keinem Zeitpunkt untersucht. Als vermutlicher Verschreibungsfehler klassifiziert wurden Änderungen der Medikation, deren Begründung schriftlich nicht deklariert war. Beabsichtigte, aber aus der Krankengeschichte nicht nachvollziehbare Änderungen wurden somit auch als vermutliche Fehler ausgewertet.

- Das Bewusstsein der Patienten über Änderungen der verschriebenen Medikamente zeigte sich in dieser Studie als sehr gering. Mögliche Gründe für dieses Resultat sind das eher höhere Durchschnittsalter der untersuchten Population (77 Jahre), sowie die Polypharmazie (Durchschnittliche Anzahl verschriebener Medikamente bei Austritt 8.0).

- Insgesamt zeigt diese Studie eine grosse Zahl nicht nachvollziehbarer, mutmasslich fälschlicher Anpassungen von Medikamentenverschreibungen im Zeitraum zwischen Spitaleintritt und Spitalaustritt. Zudem fand sich eine hohe Fehleranfälligkeit bei der Umsetzung von Verschreibungen nach Spitalaustritt auf Patientenseite. Dies Illustriert den Stellenwert des korrekten Abgleiches bei Medikamentenverschreibungen, sowie die schwierige Umsetzung von polypharmazeutischen Behandlungsplänen in einem multimorbiden, betagten Patientengut.

\section{Literatur:}

Ziaeian B, Araujo KL, Van Ness PH, Horwitz LI. Medication reconciliation accuracy and patient understanding of intended medication changes on hospital discharge. J Gen Intern Med. Nov 2012;27(11):1513-1520.

\section{Verfasser:}

Stefan Markun 